Horizons philosophiques

\title{
Montaigne et Rousseau ou le bonheur animal d'être à soi
}

\section{Philippe Pruvost}

Volume 14, numéro 1, automne 2003

$\mathrm{Au}$ risque du bonheur

URI : https://id.erudit.org/iderudit/801248ar

DOI : https://doi.org/10.7202/801248ar

Aller au sommaire du numéro

Éditeur(s)

Collège Édouard-Montpetit

ISSN

1181-9227 (imprimé)

1920-2954 (numérique)

Découvrir la revue

Citer cet article

Pruvost, P. (2003). Montaigne et Rousseau ou le bonheur animal d'être à soi. Horizons philosophiques, 14(1), 1-13. https://doi.org/10.7202/801248ar d'utilisation que vous pouvez consulter en ligne.

https://apropos.erudit.org/fr/usagers/politique-dutilisation/ 


\section{MONTAIGNE ET ROUSSEAU OU LE BONHEUR ANIMAL D'ÊTRE À SOI}

Traditionnellement, la philosophie ne promet la vie heureuse qu'à ceux qui ont accolé la pensée à la vie. L'exercice de la pensée va être à la fois le moyen d'accès à la vie heureuse et la vie heureuse elle-même. On sait que l'épicurien n'accède au bonheur que par l'élimination de la souffrance et du déplaisir. Grâce au crible de l'activité intellectuelle, l'homme découvre le plaisir : la conscience est la nécessaire accompagnatrice de toute jouissance; garder son plaisir, c'est justement savoir ne pas en abuser. Le plaisir est bien une affaire de mesure et donc de raison.

Le stoïcisme voit l'accomplissement de l'homme, sa béatitude en quelque sorte, dans la révélation que l'homme possède en lui-même une capacité exceptionnelle de "résilience», pour parler à la mode. II y a en effet un optimisme stoïcien qui s'appuie sur le fait que l'homme dispose d'une "citadelle intérieure" contre laquelle viendront se briser les inévitables vicissitudes de la vie. Bien sûr, comme l'a superbement montré Hadot 1 , le succès n'est pas garanti du premier coup, ni pour le premier venu. Avant d'en arriver à cette invulnérabilité, l'apprenti philosophe devra multiplier les exercices de penser avec lui-même. Ces "exercices spirituels" ont d'abord pour fonction de distinguer ce qui dépend de nous de ce qui n'en dépend pas. Cette opération faite - et elle n'est pas mince, car il s'agit d'un formidable renoncement,le stoïcien met à jour la ridicule importance de l'homme et l'immense modestie à laquelle il est désormais convié.

L'homme n'est pas grand-chose; il doit dans la plupart des cas se plier aux ordres du destin. Toutefois, cette réduction de l'homme n'est pas la résignation. II reste au sage stoïcien une carte à jouer, celle de l'acceptation de ce destin, se plier à la nature et à l'ordre du monde. II est donc vain de lutter; il faut accompagner le destin en travaillant justement sur le peu qui dépendra encore de nous, c'est-à-dire nos représentations. Ce qui nous affecte, ce ne sont pas les choses elles-mêmes (car il est inutile de s'opposer à l'inexorable), mais ce sont les idées que nous nous en faisons. La souffrance, le mal existent et nous n'y pouvons rien. Reste encore à ne pas leur donner l'importance qu'ils n'ont pas. En travaillant sur les représentations, le 
stoïcien se met en situation non pas de parvenir à la félicité, mais comme le dira Kant plus tard, d'être en état de pouvoir en profiter, si jamais elle arrive. Dans le stoïcisme, la vie heureuse n'est peut-être plus le produit de l'activité de l'esprit, mais du moins nous permet-elle de ne pas être anéanti par la vie.

Plus pessimistes, les positions sceptiques et cyniques ne garantissent plus qu'une vie accompagnée de réflexion soit synonyme de bonheur. Dans ces deux écoles le travail essentiellement négatif de la pensée va rendre impossible la recherche d'un état d'harmonie. Pour les sceptiques, la vérité n'existe pas et la vie n'a donc aucune justification : tout est bien ou tout est mal. L'idée d'un sens positif à la vie et la recherche d'un équilibre relève de la pure illusion. Au mieux le sage peut-il espérer trouver dans sa force d'âme la capacité d'être indifférent au monde et ainsi n'en pas souffrir. Pour les disciples de Diogène, tout est consommé; la subversion de l'esprit poussée à son extrême a tôt fait de dégonfler toutes les prétentions; tout n'est que vanités, supercheries et mensonges, y compris cela même qui permet de tout dénoncer, c'est-à-dire la pensée elle-même ou la prétendue sagesse qui devrait en découler. La pompe du raisonnable n'est qu'une immense illusion pour l'homme qui se dit plus près du chien que de l'homme. D'où cette sauvagerie, cette insociabilité de l'homme chien qui aboie et qui mord ceux qui essaient de lui parler et de lui témoigner quelque sympathie. La liberté exceptionnelle qui résulte de ce "discours vrai» est malgré tout bien amère car elle éloigne du sage les hommes, ou du moins ceux qui se prétendent tels. Pour l'homme chien, la lucidité a un prix : la solitude et l'exclusion de celui qui a "tout compris" mais qui ne coïncide plus ni avec le monde, ni avec les hommes et encore moins avec lui-même. Dupe de rien, le cynique a rompu avec tout ce qui résulte du plaisir animal d'être là, du plaisir de l'abandon à la spontanéité, naìve peut-être, du vivant.

Avec le cynisme nous parvenons au terme d'un processus où il apparaît que la vie heureuse n'est sans doute pas celle produite par la pensée. II reste au sage la lucidité qui lui tient lieu d'ultime et de bien fragile consolation. Ainsi, le bonheur relèverait de l'utopie, dans la mesure où il y aurait une outrecuidance à espérer domestiquer par la raison ce qu'il y a d'humain en nous. À moins bien sûr que devant cette incapacité radicale, on nous incite à rechercher le bonheur dans un au-delà de la vie. La promesse d'une autre vie, meilleure cette fois, devenant le seul garant d'un sens positif à notre existence. Se mettre 
en quête du bonheur, c'est donc très vite se rendre compte qu'il est inaccessible, car ce qui nous permettrait d'y accéder s'avère en être la négation. La vie heureuse du philosophe est finalement le renoncement à cette aspiration de pouvoir coïncider avec soi-même. II restera comme consolation à cette conscience malheureuse l'espérance religieuse d'une réalisation du monde des fins.

Pourtant, cette double impasse ne dissipe pas le sentiment très primitif de la légitimité de cette aspiration et quoi qu'on en dise de son accessibilité. Pour sortir de cette situation aporétique, nous invoquerons Montaigne et Rousseau, non pas comme des maîtres à penser, mais plutôt en tant que francs-tireurs de la philosophie. La trop belle familiarité de Montaigne avec ses classiques, les "académiques vierges" qu'il honorait seul dans sa tour et dont les citations incrustaient les poutres, ne doit pas trop faire illusion. La fréquentation des hommes illustres n'est peut-être pour Montaigne qu'un prétexte pour rester seul, éloigné de ses gens et de la petite vie quotidienne de son domaine. Trouvait-il plus de plaisir à observer d'en haut le grouillement quotidien de son domaine, qu'à rêvasser sur les citations latines et grecques de son plafond? La réponse n'est pas simple; chose certaine, c'est que rien ne lui échappe du haut de sa tour, fût-elle d'ivoire. II sait tout de la vie qui s'agite et il n'hésite pas à le faire savoir. Aussi le classicisme de Montaigne et son érudition savante s'accompagnent de son goût à se tenir haut, dans sa tour, comme sur son cheval, non pas pour s'éloigner, mais pour ne rien perdre du spectacle du monde. Montaigne n'est pas don Quichotte : il ne s'illusionne pas et ne se trompe pas d'époque.

La marginalité de Rousseau provient de son instinctive méfiance pour tout ce qui n'est pas médiatisé par un assentiment intérieur. II sait, lui, "le plus sociable et le plus aimant des humains"», que son malheur (et celui des autres hommes) provient de ce qui est convenu et conventionnel et non de lui-même et de sa nature. Cette certitude instinctive, dans un siècle affairé à promouvoir la raison et ses lumières comme panacées aux malheurs des hommes, ne le quittera pas. Pour Rousseau, la raison, les connaissances ne contribuent en rien à la félicité des hommes. Oser mettre en question le dogme du siècle condamnera Jean-Jacques à la marginalité, mais peut-être pas au malheur.

Chassant donc sur les marges, Montaigne et Rousseau vont en quelque sorte renouveler la problématique de la vie heureuse, en ce 
sens que l'un, comme l'autre, n'en propose nul modèle, n'en assure aucunement l'accès et n'en annonce nulle venue, mais chacun au terme d'une intime expérience va en avoir la révélation : être heureux, c'est être à soi. Or, le plus étrange de cette découverte, c'est qu'elle se fait de la même façon, dans des circonstances quasi identiques. Montaigne comme Rousseau sont renversés par un animal, un cheval, un "puissant roussin ${ }^{3}$ " pour Montaigne et un chien, "un gros chien danois ${ }^{4}$ pour Rousseau. Nos deux hommes sont à terre, commotionnés, sanglants, inconscients et tenus pour morts. Ils vont émerger progressivement de leurs états comateux et en donner un récit quasi identique. Une parenthèse ici. Cette similitude pourrait effectivement entraîner certains à faire de Rousseau un «lecteur» un peu trop fidèle de Montaigne, ce qui aurait pour effet d'alimenter encore les préventions, et elles sont grandes, de Rousseau contre le genre humain!

Dans leur récit, que nous disent ces deux hommes? Une chose finalement fort simple : l'approche de la limite extrême n'est aucunement effroyable. Rousseau éprouve "un calme ravissant, auquel chaque fois que je me le rappelle je ne trouve rien de comparable dans toute l'activité des plaisirs connus ${ }^{5}$ ». Montaigne décrit un état doux et paisible : «je n'avais affliction ni pour autrui, ni pour moi; c'était une langueur et une extrême faiblesse, sans aucune douleuro"».

Par cet incident nos deux hommes vont faire l'expérience d'un état plus profond de soi, âme ou ça, peu importe le mot. L'essentiel tient au fait que leur est révélée notre familiarité congénitale avec la mort. Certes elle n'est pas souhaitable, mais l'effroi dont on l'entoure est surfait. Pour Montaigne, le moi profond, celui qui existe au delà de toute volonté et donc de la douleur, correspond à cet état vaporeux où l'âme est comme «léchée seulement et arrosée par la molle impression des sens ${ }^{7}$ ". L'indifférence, la légèreté de l'âme ainsi libérée des pesanteurs physiques font dire à Montaigne que s'abandonner à ce mouvement eût été «une mort bien heureuse, car la faiblesse de mon discours (raisonnement) me gardait d'en rien juger, et celle du corps d'en rien sentir 8 ". La douleur, la "mort plus vive ${ }^{9}$ " ne viendront qu'après dans le retour à la vie.

Rousseau tient le même discours. II raconte cet état d'hébétude heureuse durant son long retour à pied à travers Paris. La nuit tombe : "Je marchais très bien, très légèrement, sans sentir ni douleur ni blessure ${ }^{10}$ ». Ce sont les cris de sa femme qui lui indiquent la gravité 
de son état. II n'en passera pas moins la nuit «sans connaître encore et sentir (son) mal11». Au matin seulement il découvrira ses plaies et la douleur qui les accompagne. Que suppose tout ceci, sinon que l'effroyable, du moins ce qu'on nous a présenté comme l'archétype du malheur, n'est pas ce qu'on en dit. Pour en être "revenus", Montaigne comme Rousseau nous en donnent une image apaisée. Frôler la mort fait naître un sentiment d'harmonie et de douceur. Mais ce qu'il y a de plus remarquable, c'est la manière dont les deux auteurs parviennent à cette consolation. On connaît les préventions de Rousseau sur les vaines prétentions du savoir : "Est-il temps au moment où il faut mourir d'apprendre comment on aurait dû vivre? ${ }^{12}$;; et même si plein de bonne volonté on se met à apprendre à mourir, car l'heure est proche, il confesse : "c'est précisément (cette étude) qu'on fait le moins à mon âge, on y pense à tout, hormis à cela. Tous les vieillards tiennent plus à la vie que les enfants et en sortent de plus mauvaise grâce que les jeunes gens ${ }^{13}$ ". En plus de ne pas être en phase avec la vie, la connaissance est vaine. D'abord, celle qui lui provient du commerce avec les hommes, elle n'a pas été fructueuse, loin s'en faut : "c'est une bien triste science que celle que depuis vingt ans l'expérience m'a fait acquérir : l'ignorance est encore préférable ${ }^{14}$ ». Quant à l'autre, la "savante», celle des arguties et des subtilités métaphysiques, elle n'est d'aucun poids au regard des principes adoptés par la raison et le cœur de Jean-Jacques. «Non, de vaines argumentations ne détruiront jamais la convenance que j'aperçois entre ma nature immortelle et la constitution de ce monde et l'ordre physique que j'y vois régner ${ }^{15}$ ".

Sans trop s'avancer, on peut supposer que l'accident dont est victime Rousseau et qui le met dans cet état second d'euphorie est la confirmation de ce qu'il a depuis toujours su intuitivement, à savoir l'harmonie du monde. Cette remarquable continuité des choses et du monde, aperçue d'abord dans l'innocence de l'enfance et confirmée ensuite dans les rêveries d'un vieux promeneur solitaire lui fait voir que l'homme a les dispositions pour jouir de la bonté et de la beauté naturelle du monde. L'aptitude au bonheur existe au fond de l'homme et sa naturelle bonté en est le signe.

Même attitude chez Montaigne qui a ressenti dans la proximité de la mort cette "harmonie heureuse presque tendre ${ }^{16}$ ». Montaigne va faire de ce qui apparaît finalement comme un banal accident de cheval et un "événement léger», une toute nouvelle approche de la 
mort et, partant, de la vie elle-même. Déjà dans le Livre I, l'essai vingt intitulé "Que philosopher, c'est apprendre à mourir», il avait abordé ce sujet, mais de façon assez classique. II reprenait le discours habituel sur les remèdes à employer pour conjurer cette fatalité. La pensée est à ses yeux le moyen privilégié d'apprentissage de la mort dans la mesure où elle nous permet de l'apprivoiser et de la rendre familière en lui ôtant ce qui en fait l'effroi : la soudaineté et l'étrangeté. En étant «toujours botté et prêt à partir17» nous enlevons à la mort son principal avantage qui est celui de nous surprendre. II faut, poursuit-il, la voir partout, "au broncher d'un cheval, à la chute d'une tuile, à la moindre piqûre d'épingle ${ }^{18}$ ». La mort perd de sa puissance à qui sait en défaire le masque. La pensée sert à ramener la mort à elle-même, c'est à dire à rien.

Toutefois, cette attitude, belle comme l'antique, est peut-être aussi une pose. Montaigne a beau nous répéter ses classiques, il n'est pas sûr qu'il soit lui-même convaincu, ni convaincant. Est-il bien vrai qu' «avoir vécu un jour, vous avez tout vécu ${ }^{19}$ », et que pour le sage, Thalès en l'occurrence, "le vivre et le mourir étaient indifférents 20 »? Pour le cavalier gascon il y a quelque chose de plus dans la mort, cette ennemie qui nous tient au collet et dont nous faisons mine d'ignorer la présence. Si derechef le cavalier consent à faire face, ce n'est pourtant pas par courage, c'est qu'il sait qu'il n'y a pas d'autre attitude à avoir. La mort rattrape aussi bien le couard que l'honnête homme. II faut donc se résoudre à affronter l'inexorable. "Raidissons-nous et efforçons-nous», nous enjoint-il. Reste à savoir si ce ne sont pas là que formules incantatoires, prières de philosophes pour se donner du courage, car il aimerait voir de plus près, lui le sceptique, si la formule suffit vraiment pour s'assurer d'une bonne mort. II lui vient à l'idée d'aller vérifier : «Si j'étais faiseur de livre, je ferais un registre commenté des morts diverses 21 ». Une façon de nous dire son absence de certitude en la matière. L'essai «De l'exercitation" du Livre II (chapitre 6) présente une autre approche du problème. À la différence de l'autre essai, l'auteur nous annonce dès le début qu'il ne croit plus que «les discours et l'instruction» seuls, c'est-à-dire la voie classique, "soient assez puissants pour nous acheminer jusqu'à l'action, si outre cela nous n'exerçons et formons notre âme par expérience au train auquel nous la voulons ranger22".

II demeure toutefois une difficulté. Montaigne nous dit qu'«on se peut, par usage et par expérience, fortifier contre les douleurs, la 
honte, l'indigence et tels autres accidents; mais quant à la mort, nous ne la pouvons essayer (expérimenter) qu'une fois. Nous y sommes tous apprentis quand nous y venons ${ }^{23}$ ”. Qu'à cela ne tienne et qu'ici grâce soit rendue au "puissant roussin", ce cheval fougueux qui lui fit voir de près ce que bien peu d'hommes ont pu voir : la mort ellemême. Le récit détaillé et finement analysé du fameux incident et de ce qui en découla n'est rien d'autre que "l'exercitation" de la mort elle-même. Par chance, Montaigne a suivi le chemin qui mène de vie à trépas; il a même accepté cette mort d'ailleurs déjà concédée par tous, mais miraculeusement; il en est revenu pour dire que la mort n'est pas ce qu'on en dit. Lui, pour l'avoir côtoyée, bien plus, vécu au plus profond de sa chair, sait que la mort est l'absence totale de douleurs physiques : ce savoir charnel, existentiel est aux yeux de Montaigne bien supérieur à la connaissance objective de la mort. Certes, il ne le contredit pas, la mort reste bien l'ennemi, mais un ennemi qu'on a fréquenté, ne serait-ce qu'une fois, est un ennemi différent. Fait à souligner, cette familiarité avec la mort n'est plus livresque; ici on n'attend nul secours des philosophes ou des hommes de foi. Montaigne s'est approché seul et il nous déclare du haut de sa seule expérience que "pour apprivoiser la mort", lui faire perdre la terreur qu'elle suscite, il n'est plus utile de philosopher (cela n'est pas

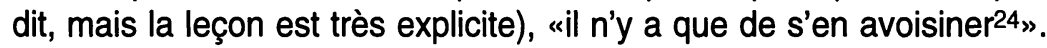
Et cette vérité là, ajoute-t-il, "ce n'est pas ma doctrine, c'est mon étude; et n'est pas la leçon d'autrui, c'est la mienne25".

Par cette brève déclaration Montaigne donne un formidable congé autant aux lumières de la foi qu'à celles de la raison. La consolation, si consolation il y a, ne peut finalement venir que de nous. L'exercitation du plus grand des périls a montré à Montaigne qu'il est vain de s'inquiéter et pathétique d'essayer de s'accrocher aux plus folles chimères. Désormais Michel de Montaigne sait de "bonne foi" que l'homme est naturellement pourvu contre la mort et ainsi contre les angoisses qu'elle suscite. L'exercitation a bien ouvert la voie d'une vraie sérénité.

II en est sorti une familiarité avec la mort, presque une tendresse, qui embrasse le principe menaçant la vie tout comme la vie elle-même, - un amour double qui, dans son indifférence morale, n'est plus obligé de "surmonter" la mort en peinant, ni de tenir la vie pour une chose indigne pour la raison qu'elle peut être anéantie. Le côté négatif de la mort n'est pas 
pour autant effacé par quelque argumentation : elle reste la destructrice, l'ennemie. Mais on peut tendre la main à cet ennemi, s'en faire un ami. II n'est pas si mauvais que nous le croyons généralement. La mort, en effet, est une possession du moi, exactement aussi ancienne que celui-ci, unique comme lui et de même nature26.

Le choc de la rencontre brutale avec l'animal a donc servi de révélateur. II a permis à des hommes, Montaigne comme Rousseau, de se rendre compte, dans un moment d'inconscience, qu'il existait une unité du monde, de la vie et de la mort. "La mort se mêle et confond partout à notre vie ${ }^{27}$ ». Forts de cette révélation, les deux auteurs s'en sont trouvés apaisés. Le malheur promis n'existerait donc pas; il ne reste alors que le bonheur d'exister. Certitude qui ne doit plus rien à la raison ni à la foi, mais à la seule expérience personnelle. Bien sûr, il faut se garder de faire des inférences "psychologisantes" entre un avant (l'accident) et un après (les conséquences). On voit tout au plus Rousseau trouver le courage de dire son fait à une flagorneuse qui l'importunait depuis un certain temps. La succession temporelle n'est pourtant pas la causalité. Quant à Montaigne, il ne dit pas ce que cela a changé dans sa vie. L'événement est d'emblée présenté comme anodin; ce qui l'est moins, c'est «'instruction que j'en ai tirée pour moi28". Certes, on l'a vu, la leçon est d'importance, mais on ne voit pas en quoi elle aurait changé la manière de vivre de Montaigne. Ce serait faire injure à la finesse de Montaigne que d'essayer d'établir des filiations causales, une clarté scolaire par exemple dans ce qui reste fondamentalement opaque, la vie elle-même. Montaigne sait bien que malgré nos prétentions, nous restons lourdement humains. La connaissance que nous pouvons tirer de nous-mêmes est celle de notre médiocrité. S'engager dans l'étude de soi ne garantit surtout pas d'y parvenir. Rien n'est simple, l'âme de l'homme est tortueuse, pleine d'inconséquence et de faiblesse. «De l'expérience que j'ai de moi, je trouve assez de quoi me faire sage, si j'étais bon écolier 29 ». On aura bien compris qu'il ne l'est pas tout à fait, bon écolier. Montaigne ne croit pas en la possibilité d'une vie ordonnée; sa vie n'est pas un modèle de cohérence : «Non seulement je trouve malaisé d'attacher nos actions les unes aux autres, mais chacune à part soi je trouve malaisé de la désigner par quelque qualité principale, tant elles sont doubles et bigarrées à divers lustres 30 ». Montaigne s'est assez coltiné avec lui-même pour connaître ses limites et démasquer ses propres 
vanités : «Le jugement tient chez moi un siège magistral, au moins il s'en efforce soigneusement; il laisse mes appétits aller leur train, et la haine et l'amitié, voire et celle que je me porte à moi-même, sans s'en altérer ni corrompre. S'il ne peut réformer les autres parties selon soi, au moins ne se laisse-t-il pas déformer à elles : il fait son jeu à part ${ }^{31}$ ».

N'est-ce pas là le maximum à espérer! La connaissance de soi est bien celle de nos limites, des portes qui nous sont closes. La chasse aux connaissances est sans fin, car une nouvelle déloge l'ancienne et ainsi de suite. Notre esprit est dans un état permanent d'agitation. «Les hommes méconnaissent la maladie naturelle de leur esprit : il ne fait que fureter et quêter, et va sans cesse tournoyant, bâtissant et s'empêtrant en sa besogne, comme nos vers de soie, et s'y étouffe. Une souris dans la poix ${ }^{32}$ ". Alors à quoi se fier, comment peut-on vivre? C'est tout simple. Selon Montaigne, il faut suivre ses inclinations naturelles. "Je donne grande autorité à mes désirs et propensions".

Vivre, c'est donc coïncider avec ce moi profond qui trace infailliblement la voie. L'animal n'a pas été que le révélateur de la vie; il en est aussi le modèle. La vie, c'est vivre sans avoir d'autre horizon que soi-même, faire l'expérience de sa propre nature sans illusion. Car il n'existe plus ni qualités ni défauts, ni d'âge meilleur qu'un autre. La maturité lui donne l'occasion d'abandonner la vie active, mais cette retraite est sans mérite. On ne fait que ce qu'on peut faire. La vie contemplative n'est possible que pour celui dont les passions sont devenues tièdes et Montaigne n'est pas prêt à jouer les vertueux. II essaie, dit-il, de vivre pour lui ce bout de vie qui lui reste. Sachant que cette vie désengagée n'est pas meilleure que l'autre, la vie active, c'est la raison pour laquelle il passera si aisément de l'une à l'autre. II sortira de sa retraite pour devenir maire de Bordeaux. Ce sont uniquement les "petites âmes" qui, ensevelies sous le poids des affaires, ne savent pas s'en démêler et ne peuvent faire ce va-et-vient. Montaigne n'est écrasé par rien; corps ou âme, plaisir ou devoir, il faut donner sa part à chaque domaine et surtout être flexible en s'abandonnant à ce que le sort aura décidé.

La retraite de Montaigne n'est pas celle de l'homme hors du temps, mais «un refuge intérieur toujours disponible, qui n'interdit pas à l'âme de donner au monde ce qu'il réclame et d'y prendre le plaisir qu'elle y trouve ${ }^{34}$ ». Cette possibilité de mise à distance garantit une 
ouverture d'esprit et une disponibilité permanente. Montaigne est toujours bien là où il est, quoi qu'il fasse. "Quand je danse, je danse; quand je dors, je dors; voire et quand je me promène solitairement en un beau verger, si mes pensées se sont entretenues des occurrences étrangères quelque partie du temps, quelque autre partie je les ramène à la promenade, au verger, à la douceur de cette solitude et à moi35».

Grâce à cette souplesse qui permet la coïncidence avec les choses et les êtres, il est à son aise, pleinement étendu dans la vie, comme dans son lit, "à la royale», comme il le dit, c'est-à-dire seul et parfaitement satisfait de l'être. Cette vie pour soi, on l'a vu, n'exclut pas le commerce avec les autres hommes, les femmes aussi, mais ce qu'il cherche dans les rencontres et surtout les voyages, c'est l'occasion de se développer lui-même et de s'épanouir encore plus. II existe toujours une réserve, une distance, sauf dans l'amitié - mais la chose est si exceptionnelle qu'on peut estimer «heureux qui avait pu rencontrer seulement l'ombre d'un ami». Montaigne a cette chance d'éprouver ce sentiment si rare "où chacun devient soi-même à travers l'autre 36 ". L'amitié permet enfin l'oubli de soi, car l'autre vous rend intégralement ce que vous lui donnez. Cet autre lui-même, Montaigne l'a, hélas, perdu. La Boétie mort, sa vie qu'il confesse avoir eu douce et aisée a perdu de sa saveur. Ces quatre années d'amitié sont tout et le reste "n'est que fumée, ce n'est qu'une nuit obscure et ennuyeuse ${ }^{37}$ ". Mais n'est-ce pas un grand bonheur d'avoir connu le bonheur?

C'est ce que Rousseau nous dit dans sa dernière Promenade. La plénitude, il l'a connue avec Madame de Warens, cette maman qui a permis à Jean-Jacques d'être enfin lui-même. Ce temps du bonheur, ce fut «le temps de ma vie où je fus moi pleinement sans mélange et sans obstacle, et où je puis véritablement dire avoir vécu38». Dans cet état de béatitude il découvre ce qu'il est et ce qu'il veut : «je fis ce que je voulais faire, je fus ce que je voulais être ${ }^{39}$ ". Hélas, Rousseau, rattrapé par la vie et ses exigences, s'aperçoit que le goût de la solitude et de la contemplation qui lui ont été révélés vont être de sérieux handicaps pour quelqu'un de sa condition. Ces moments de bonheur, il tâchera, en développant ses talents, d'en payer le prix, et ainsi de rendre ce qui lui a été donné.

Cette carrière d'homme public le précipita dans le malheur. II fut le jouet de ses bruyants et prétendus amis dont il s'aperçut avoir été 
la dupe et la victime. À l'occasion de sa chute - il tombe de moins haut que Montaigne - le promeneur solitaire et insouciant redécouvre soudainement l'extase de la coïncidence avec la vie. II connaît mieux que quiconque ses faiblesses; il les invoque, mais sans en faire des excuses. II avoue avoir "débité bien des fables ${ }^{40}$, mais avoir rarement menti. La vérité n'est une vertu que si elle ne nuit à personne, et les fables de Jean-Jacques n'ont nui à personne. La faiblesse de son âme et sa nature timide lui interdisent en outre de se poser en modèle de vertu. Jean-Jacques est ce qu'il est, rien de plus. Cette sincérité de l'innocence retrouvée et acceptée lui permet de refaire comme dans son accident l'expérience de cette pure perméabilité de l'être qui fusionne avec le monde. Le bonheur, c'est "ce petit nombre d'années aimé d'une femme pleine de complaisance et de douceur", mais un bonheur déjà gangrené par la crainte qu'il ne finisse un jour. Le vrai bonheur, l'extase, ce sont ces deux mois passés sur l'île de Saint-Pierre au milieu du lac de Bienne en Suisse. Deux mois qui auraient pu être deux ans, une éternité où Rousseau vécut, et cette fois seul ou presque, l'expérience de la pure dilatation de soi. Le bonheur consiste d'abord à ne rien faire. "Le précieux far niente fut la première et la principale de ces jouissances que je voulus savourer dans toute sa douceur41». Solitude ensuite : «je m'étais», nous dit-il, "enlacé de moi-même", en laissant là malles et caisses non déballées comme pour mieux jouir de ces instants uniques et "au lieu de ces tristes paperasses et de toute cette bouquinerie, j'emplissais ma chambre de fleurs et de foin; car j'étais alors dans ma première ferveur de botanique ${ }^{42}$ ». Oisiveté, solitude, botanique, voilà les ingrédients; il suffit de secouer et vous avez le bonheur. Le vrai bonheur n'est pas celui des petits plaisirs de la vie, mais celui procuré par le sentiment animal de notre existence. L'association du plaisir au bonheur est funeste à ce dernier car le plaisir est fugace par définition et le bonheur ne peut être cela. "Comment peut-on appeler bonheur un état fugitif qui nous laisse encore le cœur inquiet et vide, qui nous fait regretter quelque chose avant, ou désirer encore quelque chose après?43»

L'état dans lequel se trouva Rousseau à l'île de Saint-Pierre est un état de pure coïncidence où pour l'âme il n'existe ni passé ni avenir :

où le temps ne soit rien pour elle, où le présent dure toujours sans néanmoins marquer sa durée et sans aucune trace de succession, sans aucun autre sentiment de privation ni de 
jouissance, de plaisir ni de peine, de désir ni ne crainte que celui seul de notre existence, et que ce sentiment seul puisse la remplir tout entière; tant que cet état dure, celui qui s'y trouve peut s'appeler heureux, non d'un bonheur imparfait, pauvre et relatif, tel que celui qu'on trouve dans les plaisirs de la vie, mais d'un bonheur suffisant, parfait et plein, qui ne laisse dans l'âme aucun vide qu'elle sente le besoin de remplir44.

Ce que Rousseau décrit ici, c'est le sentiment animal de notre existence que l'on éprouve dans la plénitude de la coïncidence avec soi. «De quoi jouit-on dans une pareille situation? De rien d'extérieur à soi, de rien sinon de soi-même et de sa propre existence, tant que cet état dure on se suffit à soi-même comme Dieu45\%. II reste que cette harmonie et cette paix pourraient être troublées par l'extérieur. D'où la métaphore de l'île comme lieu de sérénité hors du temps et hors du monde. Toutefois, il ne faut pas que cette paix soit trop grande, car «sans mouvement la vie n'est qu'une léthargie. (...) Un silence absolu porte à la tristesse. II offre une image de la mort 46 ». Ce mouvement doit donc être régulier et doux, mais surtout il doit venir de nous-mêmes. Le mouvement de l'âme qu'est l'imagination permet "de se souvenir de soi en oubliant tous ses maux 47 ».

L'île a été l'occasion pour le rêveur qu'était Rousseau d'éprouver le sentiment d'être à soi. Moment magique, exceptionnel certes, mais l'imagination et la rêverie permettent au promeneur solitaire d'avoir, tout comme le dit Montaigne, son "arrière boutique» bien à lui, seule assurance de liberté et donc de plénitude.

\section{Philippe Pruvost Philosophie Collège de Rosemont}

1. Pierre Hadot, La citadelle intérieure, Paris, Fayard, 1992.

2. Jean-Jacques Rousseau, Les Rêveries du Promeneur solitaire, Lausanne, éditions Rencontre, 1963, p. 35

3. Michel de Montaigne, Les Essais, Paris, Arléa, 2002, p. 273.

4. Jean-Jacques Rousseau, op. cit., p. 56.

5. Jean-Jacques Rousseau, op. cit., $0,57$.

6. Michel de Montaigne, op. cit., p. 275.

7. Ibid.

8. Ibid. 
9. Ibid.

10. Jean-Jacques Rousseau, op. cit., p. 58.

11. Ibid., p. 59.

12. Jean-Jacques Rousseau, op. cit., p. 69.

13. Ibid., p. 70-71.

14. Ibid., p. 69.

15. Ibid., p. 82.

16. Hugo Friedrich, Montaigne, Paris, Gallimard, collection Tel, 2002, p. 292.

17. Michel de Montaigne, op. cit., p. 72.

18. Ibid., p. 71.

19. Ibid., p. 76.

20. Ibid., p. 78.

21. Ibid., p. 73.

22. Ibid., p. 271.

23. Ibid.

24. Ibid., p.276.

25. Ibid.

26. Hugo Friedrich, op. cit., p. 294.

27. Michel de Montaigne, op. cit., p. 785.

28. Ibid., p. 270.

29. Ibid., p. 765.

30. Ibid., p. 767.

31. Ibid., p. 766.

32. Ibid., p. 761.

33. Ibid., p. 774.

34. Hugo Friedrich, op. cit., p. 260.

35. Michel de Montaigne, op. cit., p. 789.

36. Hugo Friedrich, op. cit., p. 253.

37. Michel de Montaigne, op. cit., p. 146.

38. Jean-Jacques Rousseau, op. cit., p. 240.

39. Ibid., p. 241.

40. Ibid., p. 120.

41. Ibid., p. 128.

42. Ibid., p. 129.

43. Ibid., p. 136.

44. Ibid., p. 136-137.

45. Ibid., p. 137.

46. Ibid., p. 138.

47. Ibid., p. 139. 